Research Article

\title{
Systems Pharmacology and In Silico Docking Analysis Uncover Association of CA2, PPARG, RXRA, and VDR with the Mechanisms Underlying the Shi Zhen Tea Formula Effect on Eczema
}

\author{
Zhen-Zhen Wang $\mathbb{D}^{1,2}$ Yuan Jia, ${ }^{1}$ Kamal D. Srivastava, ${ }^{2,3}$ Weihua Huang, ${ }^{4}$ Raj Tiwari, ${ }^{2,5}$ \\ Anna Nowak-Wegrzyn, ${ }^{6,7}$ Jan Geliebter, ${ }^{2,5}$ Mingsan Miao $\mathbb{D}^{1},{ }^{1}$ and Xiu-Min Li $\mathbb{D}^{2,5}$ \\ ${ }^{1}$ Academy of Chinese Medical Science, Henan University of Chinese Medicine, Zhengzhou 450046, China \\ ${ }^{2}$ Department of Microbiology \& Immunology, New York Medical College, New York 10595, USA \\ ${ }^{3}$ General Nutraceutical Technology LLC, Elmsford, New York 10523, USA \\ ${ }^{4}$ Department of Pathology, New York Medical College, New York 10595, USA \\ ${ }^{5}$ Department of Otolaryngology, School of Medicine, New York Medical College, New York 10595, USA \\ ${ }^{6}$ Department of Pediatrics, New York University Langone Health, New York, NY 10029, USA \\ ${ }^{7}$ Department of Pediatrics, Gastroenterology and Nutrition, Collegium Medicum, University of Warmia and Mazury, \\ Olsztyn 10-561, Poland
}

Correspondence should be addressed to Mingsan Miao; miaomingsan@126.com and Xiu-Min Li; xiumin_li@nymc.edu

Received 2 July 2020; Revised 18 March 2021; Accepted 27 March 2021; Published 17 May 2021

Academic Editor: Nasiara Karim

Copyright $(2021$ Zhen-Zhen Wang et al. This is an open access article distributed under the Creative Commons Attribution License, which permits unrestricted use, distribution, and reproduction in any medium, provided the original work is properly cited.

Eczema is a complex chronic inflammatory skin disease impacted by environmental factors, infections, immune disorders, and deficiencies in skin barrier function. Shi Zhen Tea (SZT), derived from traditional Chinese medicine Xiao-Feng-San, has shown to be an effective integrative therapy for treating skin lesions, itching, and sleeping loss, and it facilitates reduction of topical steroid and antihistamine use in pediatric and adult patients with severe eczema. Yet, its active compounds and therapeutic mechanisms have not been elucidated. In this study, we sought to investigate the active compounds and molecular mechanisms of SZT in treating eczema using systems pharmacology and in silico docking analysis. SZT is composed of 4 medicinal herbs, Baizhu (Atractylodis macrocephalae rhizome), Jingjie (Schizonepetae herba), Kushen (Sophorae flavescentis radix), and Niubangzi (Arctii fructus). We first identified 51 active compounds from SZT and their 81 potential molecular targets by high-throughput computational analysis, from which we identified 4 major pathways including Th17 cell differentiation, metabolic pathways, pathways in cancer, and the PI3K-Akt signaling pathway. Through network analysis of the compound-target pathway, we identified hub molecular targets within these pathways including carbonic anhydrase II (CA2), peroxisome proliferator activated receptor $\gamma$ (PPAR $\gamma$ ), retinoid X receptor $\alpha$ (RXRA), and vitamin D receptor (VDR). We further identified top 5 compounds including cynarine, stigmasterin, kushenol, $\beta$-sitosterol, and (24S)-24-propylcholesta-5-ene-3 $\beta$-ol as putative key active compounds on the basis of their molecular docking scores with identified hub target proteins. Our study provides an insight into the therapeutic mechanism underlying multiscale benefits of SZT for eczema and paves the way for developing new and potentially more effective eczema therapies.

\section{Introduction}

Eczema, characterized by itchy, scaly, erythematous, and oozing skin, is the most common chronic inflammatory skin disorder, affecting $10-20 \%$ of children and $1-3 \%$ of adults
$[1,2]$. In addition, allergic diseases such as allergic rhinitis, food allergy, and asthma are frequent comorbidities in moderate-to-severe eczema patients. Eczema severely impacts the life quality of patients and exerts tremendous financial and social burden [3]. In the United States, direct 
economic costs for the treatment of atopic eczema reached approximately $\$ 3.8$ billion per year in 2015 [4]. Eczema was ranked first in 2010 global "skin disease burden," which takes into account severe health loss, including psychological, social, and financial consequences [5]. The pathogenesis of eczema is complicated and is associated with genetic and environmental factors [6]. Severe eczema often fails to respond adequately to therapies specifically targeting a single cytokine or inflammatory mediator. To date, there is no effective cure for eczema, although some treatments can temporarily relieve skin symptoms and reduce inflammation [7]. Thus, the development of new treatments to effectively regulate the immune system and rebuild the skin barrier is an unmet need.

Traditional Chinese Medicine (TCM) has demonstrated significant success in treating complex diseases by regulating multiple pathways working to return dysfunctional organ systems back to normal [8]. Over the past 20 years, multiple studies have reported the efficacy of TCM for the treatment of eczema without serious adverse effects $[9,10]$. For example, composite poria granules have shown clinical efficacy in eczema treatment [11]. Recently, Run-Zao-ZhiYang capsules (RZZYC) have been reported to provide symptom relief, maintain long-term remission, and improve quality of life, with fewer recurrences [12]. In addition, the well-known famous TCM formula Xiao-FengSan (XFS) has been used for the treatment of skin disorders since the ancient Ming Dynasty in China [13]. It was reported to be one of the top 5 most commonly prescribed herbal formulas for eczema (total prescriptions $=381,282$ ) in Taiwan [14]. We developed Shi Zhen Tea (SZT), a derivative of XFS that has been successfully used in the US as integrative eczema therapy [8]. It is comprised of four medicinal herbs, Sophorae flavescentis radix (Kushen, K), Schizonepetae herba (Jingjie, J), Arctii fructus (Niubangzi, $\mathrm{N}$ ), and Atractylodis macrocephalae rhizoma (Baizhu, B), which display strong ability of dispersing cold, dispelling wind, and dehumidification. It showed efficacy in treating moderate-to-severe eczema in both children and adults [15]. Disease severity scores decreased in all patients, and most patients stopped taking steroids or antihistamines after the 3-month treatment. However, as with most traditional medicines, its mechanisms of action are elusive, due to presence of multiple, complex compounds, and their metabolites. By combining discovery of new actionable disease targets and isolation of active compounds, the systems pharmacology approach together with highthroughput computational analysis provides a powerful tool to study mechanisms underlying TCM function. A growing body of evidence [16-18] suggests that the use of systems pharmacology can lead to an integrated understanding of drug action on the human interactome which can be used for drug discovery and pharmacological analysis of multiple agents on complex diseases along with prediction of adverse effects. Recently, certain classical Chinese prescriptions, such as Huo-Xiang-Zheng-Qi formula [19], Dan-Shen formula [20], Xiao-Zheng-Fang formula [21], and Bu-Fei-Yi-Shen formula [22], as well as antiasthma herbal medicine intervention (ASHMI), a formula previously developed by us [23], have been analyzed and evaluated by systems pharmacology.

The current study seeks to uncover the therapeutic mechanisms' underlying efficacy of SZT on eczema using systems pharmacology, through combined evaluation of absorption, distribution, metabolism, and excretion (ADME), compound feature mapping, drug target mining, target enrichment network and pathway analyses, and in silico molecular docking. The whole workflow of the study is shown in Figure 1. Results of these analyses will contribute to the understanding of how SZT formula functions, on the molecular level, in the treatment of eczema, providing the rationale and tools to improve the formulation for better TCM treatment efficacy.

\section{Materials and Methods}

2.1. Building of the Compound Database. The SZT formula is composed of four Chinese herbs: Sophorae flavescentis radix (Kushen, K), Schizonepetae herba (Jingjie, J), Arctii fructus (Niubangzi, N), and Atractylodis macrocephalae rhizoma (Baizhu, B). The compounds of these four herbs were collected from the TCM Systems Pharmacology (TCMSP) database [24].

2.2. Active Compound Prediction by Oral Bioavailability and Drug-Likeness Evaluation. Oral bioavailability (OB), one of the vital properties of drugs, is the percentage that is absorbed into the system from an orally administered dose of drugs. The compounds with $\mathrm{OB} \geq 30 \%$ (20\% variability making threshold up to 50\%) in TCMSP were selected as active compounds considering the average of $\mathrm{OB}$ (30\% with $10 \%-50 \%$ variability) in clinical studies [23].

Drug-likeness (DL) is the similarity of a given compound with the physiochemical or/and structural properties of existing drugs, which have been used to evaluate a drug's potential early in the process of discovery. A Tanimoto coefficient was developed to predict the DL value of compounds [25]. The formula is as follows:

$$
f(A, B)=\frac{A \cdot B}{|A|^{2}+|B|^{2}-A \cdot B},
$$

where $A$ represents the molecular properties of herb compounds and $B$ represents the average molecular descriptor of molecules from the DrugBank database (drugbank.ca). $\mathrm{DL} \geq 0.18$ was determined as the criterion to select potential active compounds, where 0.18 was the mean value of $\mathrm{DL}$ index in DrugBank [26].

2.3. Compound Feature Mapping. The pharmacological activities of a compound are directly related to their physicochemical properties. The physicochemical parameters included in Lipinkski's Rule of Five, including molecular weight, lipohydro partition coefficient, hydrogen donor and acceptor, and rotatable bonds, have been used to evaluate druggability of compounds [27]. Thus, estimation of relevant physicochemical properties of active compounds may provide information about their drug likeness. Thus, the 


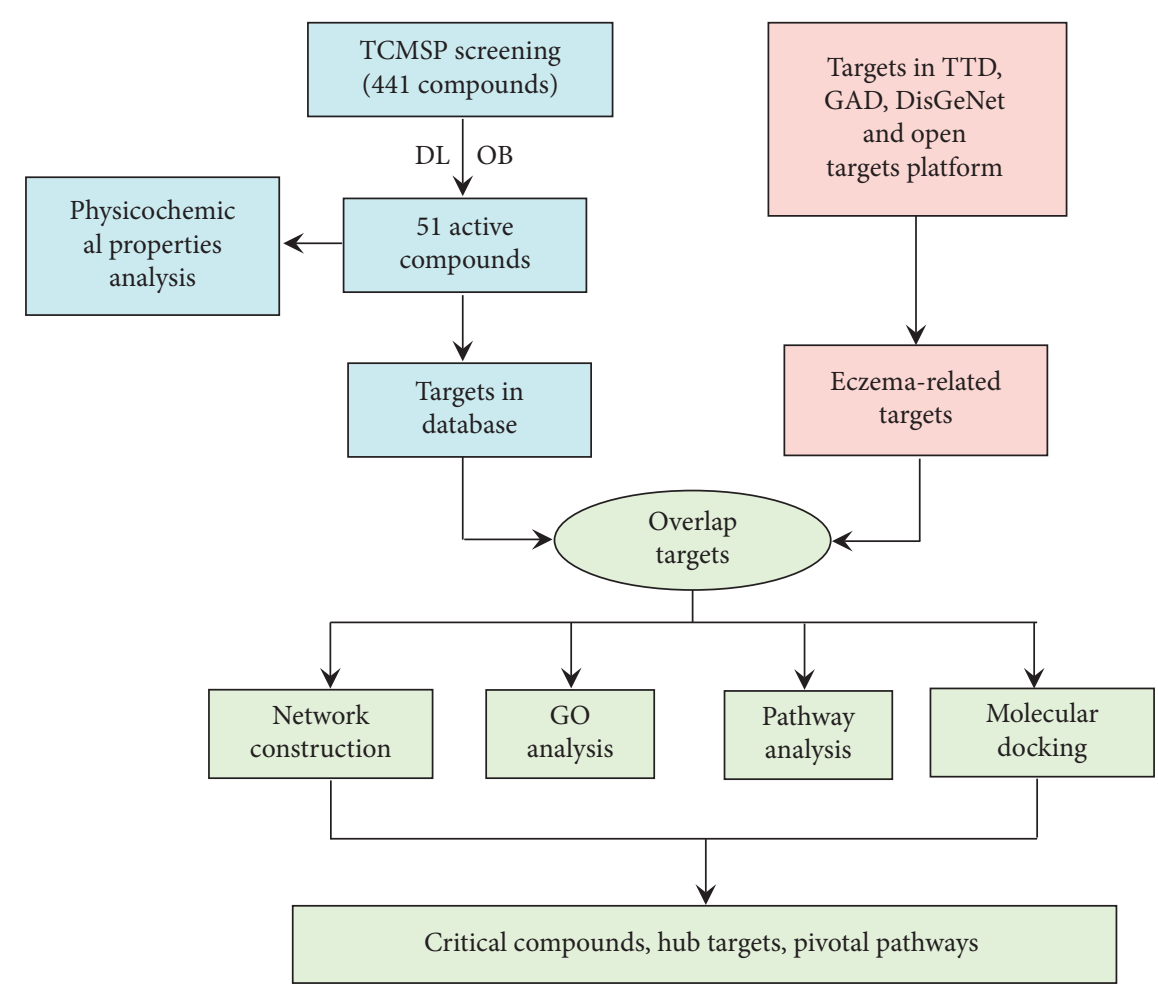

FIgURE 1: The workflow of systems pharmacology used for analysis of SZT formula for eczema treatment. TCMSP: TCM Systems Pharmacology; OB: oral bioavailability; DL: drug-likeness; TTD: therapeutic target database; GAD: genetic association database; and GO: gene ontology. First, 51 active compounds of formula were selected from the TCMSP database. Their biological targets were further collected from several reliable databases. Meanwhile, physicochemical property analysis was conducted to sum up common properties of active compounds in different physicochemical parameters. Similarly, the targets involved in the pathological process of eczema were collected from related databases including TTD, GAD, DisGeNet, and Open Targets Platform. With biological targets of compounds and related targets of eczema, their shared targets are chosen as potential therapeutic targets of SZT for eczema. Then, network construction, GO analysis, pathway analysis, and molecular docking are used to reveal the critical compounds from the SZT, the hub targets, and pivotal regulated pathway of SZT for eczema treatment.

compound feature mapping method [23] was applied to visualize all active compounds from SZT according to the following six physicochemical properties, molecular weight (MW), hydrogen bond donors (nHDon), hydrogen bond acceptor (nHAcc), octanol/water partition coefficient $($ AlogP), topological polar surface area (TPSA), and number of rotatable bonds (RBN). All six properties were analyzed by principal component analysis (PCA), where six-dimensional data were reduced to two-dimensional data with acceptable former descriptor variation in order to best display distribution of active ingredients in chemical space.

2.4. Target Fishing and Classification. First, a simplified molecular-input line-entry system (SMILES) of potential active compounds was prepared by ALOGPS 2.1 program (vcclab.org/web/alogps/). Target predictions were conducted using the SMILES of potential compounds through several published databases, including TCMSP [24], HitPick (mips.helmholtzmuenchen.de/hitpick/), Swiss Target Prediction [28] (swisstargetprediction.ch/), Similarity Ensemble Approach (SEA, sea.bkslab.org/) [29], PubChem (pubchem.ncbi.nlm.nih.gov/) [30], and DrugBank [31]. All the predicted targets obtained from these databases were mined in prevailing databases as follows to select targets for eczema: therapeutic target database
(TTD, db.idrblab.net/ttd/) [32], genetic association database (GAD, geneticassociationdb.nih.gov/), DisGeNet (disgenet.org/) [33], and Open Targets Platform (targetvalidation.org/) [34]. Selected targets were finally mapped to the UniProt Database (uniprot.org/) [35] for normalization.

2.5. Gene Ontologyand Pathway Analysis. Target enrichment gene ontology (GO) and pathway analyses lead us to gain mechanistic insight from the molecular level into biological function level. GO was introduced by mapping targets to the DAVID database (david.ncifcrf.gov/) [36]. The GO biological process terms with false discovery rate $(\mathrm{FDR})<0.01$ were selected. Pathways were obtained by mapping targets to KOBAS 3.0 (kobas.cbi.pku.edu.cn) [37]. The significant pathways with FDR $<0.01$ were selected.

2.6. Network Construction and Analysis. With predicted targets of compounds and significant pathways, we built two biological networks using Cytoscape (v3.2.1). The compound-target-disease (C-T-D) network, containing active compounds, their related targets, and disease, provides general information about the pharmacological mechanism of SZT formula in the molecular level. The principal 
pathways, extracted with its related targets and active compounds, link the pathways to candidate compounds through targets and further interweave a compound-targetpathway-disease (C-T-P-D) network. The properties of these two networks were validated by NetworkAnalyzer [38], a plugin of Cytoscape.

2.7. Molecular Docking. To explore the binding modes and offer more insights into the interaction between molecular targets and compounds, molecular docking was performed on hub targets and all related bioactive compounds by AutoDock Vina [39]. Protein crystal structures with excellent resolution were downloaded from RCSB protein data bank (rcsb.org/) [40]. The structures of the ligand were directly downloaded from PubChem (pubchem.ncbi.nlm.nih.gov/) [30] without further optimization. Proteins and compounds were prepared by AutoDockTools (v1.5.6) [41]. The three-dimensional molecular graphics were prepared by the PyMOL system [42] (pymol.org) and Discovery Studio [43]. Generally, all hydrogens and Gasteriger charges were added to each molecule. Docking areas and Autogrid parameters were set based on the binding pockets of proteins. The lowest binding energy was selected and illustrated as the best binding conformation.

\section{Results and Discussion}

3.1. Active Compounds Obtained from SZT. In total, 441 compounds from the four herbs of SZT were obtained from TCM Systems Pharmacology (TCMSP), of which 159 were from Jingjie, 113 from Kushen, 55 from Baizhu, and 114 from Niubangzi. Such complexity of SZT makes it difficult to explore its dominant compounds and uncover the biological mechanism of formula. Thus, it is necessary to determine potentially active ingredients' database from the SZT formula. Many compounds found in TCM, while being biologically active, may lack the absorption and bioavailability necessary to overcome barriers during the oral administration process and reach the targets, making it difficult to investigate the mechanisms of herbs. Poor oral bioavailability (OB) may result in low efficacy and cause a new drug to fail in clinical trials. High $\mathrm{OB}$ is a critical parameter to evaluate the possibility of a molecule becoming a drug [44]. We used oral bioavailability (OB) and drug-likeness (DL) as criteria to select the compounds as follows. $\mathrm{OB} \geq 30 \%$ and $\mathrm{DL} \geq 0.18$ were used as threshold to narrow down the compound scope, based on the theory that drugs require proper physicochemical properties to cross various barriers and play their pharmacological effects. This threshold was determined on account of not only extracting sufficient information from formula but also keeping the balance of active compound numbers from different herbs.

As a result, 51 active compounds from 441 were selected for further analysis. Abbreviation, chemical name, molecular structure, property, and species of each active compound are listed in Table 1, which included flavonoid (25.5\%), alkaloid (25.5\%), phenylpropanoid (15.7\%), terpene (13.7\%), steroid $(11.7 \%)$, and others (7.9\%). Selected compounds with satisfactory $\mathrm{OB}$ and $\mathrm{DL}$ value were assumed as potential active compounds. Among them, 25 compounds (OB $\geq 50 \%$ and $\mathrm{DL} \geq 0.18$ ) are from 113 compounds of herb Kushen. For example, matrine $(\mathrm{K} 15, \mathrm{OB}=63.77 \%$ and $\mathrm{DL}=0.25)$, one of the major tetracycloquinolizindine alkaloids, was reported to exhibit anti-inflammatory [45], antiviral [46], and antiallergic properties [47]. Flavone norkurarinol (K2, $\mathrm{OB}=51.28 \%$ and $\mathrm{DL}=0.64)$ showed various biological activities such as antioxidant, antibacterial, anti-influenza, and anti-inflammatory activities [48]. For herb Jingjie, 11 of 159 compounds met the filter criteria $(\mathrm{OB} \geq 30 \%$ and $\mathrm{DL} \geq 0.18)$. Luteolin $(\mathrm{J} 1, \mathrm{OB}=36.16 \%$ and $\mathrm{DL}=0.25)$, a flavone present in many herbs, has been proven to inhibit inflammation responses [49]. Quercetin $(\mathrm{J} 2, \mathrm{OB}=46.43 \%$ and $\mathrm{DL}=0.28)$, a well-known free-radical scavenger, can stimulate the immune system by decreasing the production of proinflammatory cytokines, suppressing IL-4 production, improving Th1/Th2 balance, and restraining the formation of antigen-specific IgE antibody [50]. In addition, phytosterols, such as $\beta$-sitosterol $(\mathrm{J} 3, \mathrm{OB}=36.91 \%$ and $\mathrm{DL}=0.75)$, sitosterol $(\mathrm{J} 4, \mathrm{OB}=36.91 \%$ and $\mathrm{DL}=0.75)$, and stigmasterol ( 5 , $\mathrm{OB}=43.83 \%$ and $\mathrm{DL}=0.76)$ have been the subject of increased interest for potential treatment of atopic dermatitis (AD) to replace steroid therapy [51]. For herb Niubangzi, 8 active compounds from 114 were identified to show $\mathrm{OB} \geq 30 \%$ and $\mathrm{DL} \geq 0.18$. Especially, arctiin (N2, $\mathrm{OB}=34.45 \%$ and $\mathrm{DL}=0.84$ ), one of the major active ingredients, can be biotransformed to its more potent aglycone arctigenin by intestinal microbiota [52]. Cynarin (N8, $\mathrm{OB}=31.75 \%$ and $\mathrm{DL}=0.68)$, an ester of quinic acid and two molecules of caffeic acid, have been proved to display good antioxidant, antiradical, and anticholinergic effects [53]. Moreover, $\beta$-sitosterol $(\mathrm{J} 3, \mathrm{~N} 3, \mathrm{OB}=36.91 \%$ and $\mathrm{DL}=0.75)$ and supraene $(\mathrm{J} 6, \mathrm{~N} 5, \mathrm{OB}=33.55 \%$ and $\mathrm{DL}=0.42)$ are shared active compounds by both Niubangzi and Jingjie. For herb Baizhu, 7 active compounds from 55 ingredients were selected to meet the criterion $(\mathrm{OB} \geq 30 \%$ and $\mathrm{DL} \geq 0.18)$. Among them, $\alpha$-amyrin $(\mathrm{B} 3, \mathrm{OB}=39.51 \%$ and $\mathrm{DL}=0.76)$, a pentacyclic triterpene, shows potential anti-inflammatory effects [54]. In particular, it significantly suppresses the scratching behavior in a mouse model of pruritus by inhibiting degranulation of mast cell [55], beneficial for skin lesions in eczema.

3.2. Compound Feature Mapping Based on Physicochemical Parameters. The drug-like physicochemical properties of active compounds were investigated by analyzing six common drug-associated physicochemical parameters including molecular weight (MW), hydrogen bond donors (nHDon), hydrogen bond acceptor (nHAcc), octanol/water partition coefficient (AlogP), topological polar surface area (TPSA), and number of rotatable bonds (RBN). Principal component analysis (PCA) was applied to display the distribution of active compounds in chemical space considering all six physicochemical parameters. The first principle component (PC1) displays the direction of maximal variance which takes $51.92 \%$ of total variance, and PC2 represents the second largest variations that account for 37.95\% (Figure 2). These two principle components account for $89.87 \%$ of the 
TABLE 1: Basic information of active compounds from SZT. F, A, P, T, S, and O stand for flavonoid, alkaloid, phenylpropanoid, terpene, steroid, and others, respectively.

\begin{tabular}{|c|c|c|c|c|c|c|c|}
\hline Herb & Comp. ID & Comp. name & Mol. Wt. & O.B. & D.L. & Structure & Sp. \\
\hline Kushen & $\mathrm{K} 1$ & kushenolJ_qt & 286.3 & 50.86 & 0.24 & & $\mathrm{~F}$ \\
\hline Kushen & K2 & Norkurarinol & 442.55 & 51.28 & 0.64 & & $\mathrm{~F}$ \\
\hline Kushen & $\mathrm{K} 3$ & KushenolJ & 580.59 & 51.39 & 0.74 & nof & $\mathrm{F}$ \\
\hline Kushen & $\mathrm{K} 4$ & $(+)$-Lupanine & 248.41 & 52.71 & 0.24 & & A \\
\hline Kushen & K5 & Norartocarpetin & 286.25 & 54.93 & 0.24 & & $\mathrm{~F}$ \\
\hline Kushen & K6 & Sophranol & 264.41 & 55.42 & 0.28 & & A \\
\hline Kushen & K7 & $(+)$-Lehmannine & 246.39 & 58.34 & 0.25 & & A \\
\hline Kushen & K8 & $(+)$-Allomatrine & 248.41 & 58.87 & 0.25 & & A \\
\hline Kushen & K9 & Sophoridine & 248.41 & 60.07 & 0.25 & & A \\
\hline Kushen & K10 & Leachianone,g & 356.4 & 60.97 & 0.4 & & $\mathrm{~F}$ \\
\hline Kushen & K11 & Isosophocarpine & 246.39 & 61.57 & 0.25 & & A \\
\hline Kushen & K12 & Anagyrine & 244.37 & 62.01 & 0.24 & & A \\
\hline Kushen & K13 & Leontalbinine & 246.39 & 62.08 & 0.25 & & A \\
\hline Kushen & K14 & Lehmanine & 246.39 & 62.23 & 0.25 & & A \\
\hline Kushen & K15 & Matrine & 248.41 & 63.77 & 0.25 & & A \\
\hline Kushen & K16 & Sophocarpine & 246.39 & 64.26 & 0.25 & & A \\
\hline Kushen & K17 & 13,14-Dehydrosophoridine & 246.39 & 65.34 & 0.25 & & A \\
\hline Kushen & K18 & Inermin & 284.28 & 65.83 & 0.54 & & $\mathrm{P}$ \\
\hline Kushen & K19 & cis-Dihydroquercetin & 304.27 & 66.44 & 0.27 & & $\mathrm{~F}$ \\
\hline Kushen & K20 & Isomatrine & 248.41 & 68.68 & 0.25 & & A \\
\hline Kushen & K21 & Formononetin & 268.28 & 69.67 & 0.21 & & $\mathrm{~F}$ \\
\hline Kushen & K22 & $\mathrm{K} 22$ & 256.27 & 71.12 & 0.18 & & $\mathrm{~F}$ \\
\hline Kushen & K23 & Inermine & 284.28 & 75.18 & 0.54 & & $\mathrm{P}$ \\
\hline Kushen & K24 & Phaseolin & 322.38 & 78.2 & 0.73 & & $\mathrm{P}$ \\
\hline Kushen & K25 & Glyceollin & 338.38 & 97.27 & 0.76 & & $\mathrm{P}$ \\
\hline Jingjie & $\mathrm{J} 1$ & Luteolin & 286.25 & 36.16 & 0.25 & & $\mathrm{~F}$ \\
\hline Jingjie & $\mathrm{J} 2$ & Quercetin & 302.25 & 46.43 & 0.28 & & $\mathrm{~F}$ \\
\hline Jingjie & $\mathrm{J} 3$ & beta-Sitosterol & 414.79 & 36.91 & 0.75 & & S \\
\hline Jingjie & $\mathrm{J} 4$ & Sitosterol & 414.79 & 36.91 & 0.75 & & S \\
\hline Jingjie & $\mathrm{J} 5$ & Stigmasterol & 412.77 & 43.83 & 0.76 & & S \\
\hline Jingjie & J6 & Supraene & 410.8 & 33.55 & 0.42 & YMn & $\mathrm{T}$ \\
\hline
\end{tabular}


TABLE 1: Continued.

\begin{tabular}{|c|c|c|c|c|c|c|c|}
\hline Herb & Comp. ID & Comp. name & Mol. Wt. & O.B. & D.L. & Structure & Sp. \\
\hline Jingjie & $\mathrm{J} 7$ & Diosmetin & 300.28 & 31.14 & 0.27 & & $\mathrm{~F}$ \\
\hline Jingjie & $\mathrm{J} 8$ & Campest-5-en-3-beta-ol & 400.76 & 37.58 & 0.71 & & S \\
\hline Jingjie & J9 & J9 & 302.3 & 47.74 & 0.27 & & $\mathrm{~F}$ \\
\hline Jingjie & $\mathrm{J} 10$ & Schizonepetoside B & 330.42 & 31.02 & 0.28 & & $\mathrm{~T}$ \\
\hline Jingjie & J11 & Schkuhrin I & 420.5 & 54.45 & 0.52 & & $\mathrm{O}$ \\
\hline Niubangzi & N1 & Neoarctin A & 742.88 & 39.99 & 0.27 & & $\mathrm{P}$ \\
\hline Niubangzi & N2 & Arctiin & 534.61 & 34.45 & 0.84 & & $\mathrm{P}$ \\
\hline Niubangzi & N3 & beta-Sitosterol & 414.79 & 36.91 & 0.75 & & $S$ \\
\hline Niubangzi & N4 & Kaempferol & 286.25 & 41.88 & 0.24 & & $\mathrm{~F}$ \\
\hline Niubangzi & N5 & Supraene & 410.8 & 33.55 & 0.42 & and & $\mathrm{T}$ \\
\hline Niubangzi & N6 & beta-Carotene & 536.96 & 37.18 & 0.58 & תים & $\mathrm{T}$ \\
\hline Niubangzi & N7 & N7 & 386.48 & 52.3 & 0.48 & & $\mathrm{P}$ \\
\hline Niubangzi & N8 & Cynarin(e) & 516.49 & 31.76 & 0.68 & & $\mathrm{P}$ \\
\hline Baizhu & $\mathrm{B} 1$ & $\mathrm{~B} 1$ & 276.41 & 35.95 & 0.21 & & $\mathrm{~T}$ \\
\hline Baizhu & B2 & B2 & 428.82 & 36.23 & 0.78 & & S \\
\hline Baizhu & B3 & $\alpha$-Amyrin & 426.8 & 39.51 & 0.76 & & $\mathrm{~T}$ \\
\hline Baizhu & B4 & $3 \beta$-Acetoxyatractylone & 274.39 & 54.07 & 0.22 & & $\mathrm{~T}$ \\
\hline Baizhu & B5 & B5 & 355.44 & 60.31 & 0.31 & & $\mathrm{O}$ \\
\hline Baizhu & B6 & B6 & 312.39 & 62.4 & 0.22 & & $\mathrm{O}$ \\
\hline Baizhu & B7 & B7 & 356.45 & 63.37 & 0.3 & & $\mathrm{O}$ \\
\hline
\end{tabular}

K22: (2R)-7-hydroxy-2-(4-hydroxyphenyl)chroman-4-one; J9: 5,7-dihydroxy-2-(3-hydroxy-4-methoxyphenyl)chroman-4-one; N7: (3R,4R)-3,4-bis[(3,4dimethoxyphenyl)methyl]oxolan-2-one; B1: 8ß-ethoxy atractylenolide III; B2: (24S)-24-propylcholesta-5-ene-3beta-ol; B5: 14-acetyl-12-senecioyl2E,8E,10E-atractylentriol; B6: 12-senecioyl-2E,8E,10E-atractylentriol; B7: 14-acetyl-12-senecioyl-2E,8Z,10E-atractylentriol.

total variances, indicating that they sufficiently represent the six physicochemical features of these compounds. Figure 2 illustrates the relatedness distribution of the active compounds. Most of the active compounds from SZT are clustered in the red circled area, indicating they have similar physicochemical features. It is noted that the binding of compounds to their targets are up to the structure and property of compounds. The result implies that the targets of these active compounds, derived from different herbs but with similar physicochemical properties, may be associated and overlapping.

3.3. Potential Target Fishing. Establishing compound-target interactions and enriching those in signaling pathways become increasingly necessary for explicating the mechanism of drug action and their underlying pharmacological effects. The targets of 51 active compounds were fished by several popular databases, including HitPick, Swiss Target Prediction, Similarity Ensemble Approach (SEA), PubChem, and DrugBank. The integrated targets were further mined in targets selected from the therapeutic target database (TTD), genetic association database (GAD), DisGeNet, and Open Targets Platform with terms "eczema" and "atopic dermatitis." Finally, a total of 81 potential targets were screened out for 51 active compounds with 327 interactions. Most compounds were found to interact with more than one target for eczema, which implies comprehensive regulation and extensive pharmacological actions of SZT. For example, phaseolin (K24) from Kushen interacts with 15 potential targets related to eczema, while kaempferol (N4) from Niubangzi links to 12 potential targets. Among these targets, vascular endothelial growth factor A (VEGFA) [56], epidermal growth factor receptor (EGFR) [57], and interleukin 2 (IL-2) [58] are believed to play crucial roles during the 


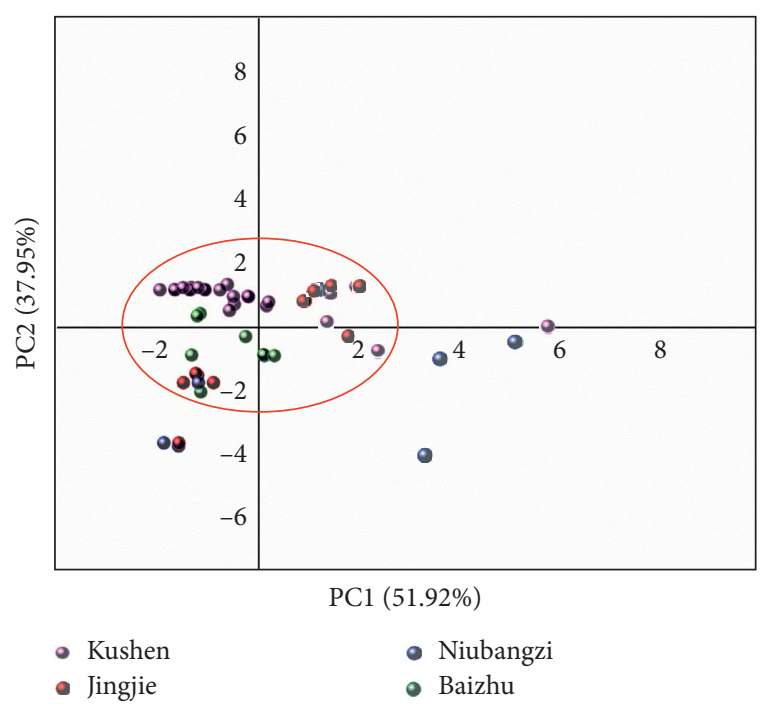

Figure 2: Chemical space distribution of active components present in Kushen, Jingjie, Niubangzi, and Baizhu, based on principal component analysis of their drug-related physicochemical properties. Active compounds in the red circle display a similar physiochemical property.

pathogenesis of $\mathrm{AD}$. $\beta$-sitosterol (N3, J3) from both Niubangzi and Jingjie targets 11 proteins, among which peroxisome-proliferator-activated receptor (PPAR) family and retinoic-acid-receptor-related orphan receptors (RORs) might be the main regulated targets of $\beta$-sitosterol [59]. RORs regulate the function of specific subsets of T cells and innate lymphoid cells, which are key drivers of inflammatory disease in barrier tissues [60]. Moreover, the retinoid X receptor (RXR) is an intriguing and essential member of nuclear receptors activated by 9 -cis retinoic acid to regulate cell differentiation, metabolism, and cell apoptosis [61]. The strong reduction of retinoid signaling and retinoid concentration in affected as well as nonaffected skin of individuals with $\mathrm{AD}$ has been discovered, compared to healthy individuals [62]. A selective activator of RAR or RXR may influence the retinoid signaling in skin, and RORA and RORC have been reported to be a potential critical therapeutic target for treating skin diseases of inflammatory etiology [63].

C-T-D network construction is used to display the active compounds and potential targets of SZT for eczema treatment.

3.3.1. Network Analysis. Active compound and target data extracted above were employed to construct a C-T-D network containing 129 nodes and 406 edges, involving 47 active compounds and 81 potential targets. Four active compounds $\mathrm{K} 11, \mathrm{~K} 25, \mathrm{~B} 5$, and B6 shared no targets with eczema and were, thus, excluded. The network demonstrates multi-interactions between compounds and targets (Figure 3). The diamonds and circles represent active compounds from SZT and their potential targets, respectively. Of 81 targets, Kushen, Jingjie, Niubangzi, and Baizhu recognized 52, 35, 43, and 19 targets, respectively. To evaluate the importance of each node in this network, the degree of each node was defined as the number of edges connected with this node, representing the influence of the node. The network average degree of node was 6.3.

3.3.2. Target Analysis. Most of the targets from our network construction are closely associated with the pathogenesis of eczema. We believe that targets with highest degree are the most vital ones regulated by SZT, thus chosen as hub targets for further analysis. Interestingly, carbonic anhydrase II (CA2) had the largest degree, hit by 17 compounds ( 5 of Kushen, 3 of Niubangzi, 7 of Jingjie, and 1 of Baizhu). $\mathrm{CA} 2$ monitors $\mathrm{pH}$ regulation, water transport, and hydration of $\mathrm{CO}_{2}$, which is related to diverse diseases such as glaucoma, tumor, epilepsy, and diabetes [64]. CA2 inhibitors, such as acetazolamide and brinzolamide, have been developed and established to treat glaucoma, seizure disorder, and acute mountain sickness [65]. It was reported that CA2 was upregulated by Th2 cytokines in lesion skin of $\mathrm{AD}$ patients, which caused a $\mathrm{pH}$ rise in skin [66]. The higher surface $\mathrm{pH}$ may affect the integrity and cohesion of skin and compromise barrier function of skin in $\mathrm{AD}$ [67]. Thus, the CA2 inhibitor may relieve a series of symptoms in dermatitis triggered by itching [68]. Moreover, VEGFA was targeted by 12 active compounds ( 6 of Kushen, 4 of Jingjie, and 2 of Niubangzi). VEGFA displays a key role in vascular permeability, vasodilation, and angiogenesis. VEGFA can be secreted by various immunocytes in inflamed skin, such as mast cells, macrophages, eosinophils, basophils, and Th17 cells, which appear critical not only in lymphatic vessel expansion but also in antigen clearance and inflammation resolution through enhancement of lymphangiogenesis [56]. Meanwhile, 12 compounds from SZT were found to interact with PPARG. PPARG belongs to a subfamily of nuclear hormone receptors and plays a distinct physiological role in regulating expression of genes involved in cellular proliferation, specific components of the Th2 inflammatory pathway, and maintenance of the skin barrier [69].

Gene ontology (GO) and pathway analysis reveal potential regulation of SZT in the immune, inflammatory, and metabolic processes.

Utilizing the identified targets as an enriched gene set, we employed gene-set enrichment analysis to find GO biological process terms in the DAVID database and pathways in the KOBAS database. The top 15 biological process GO terms with False Discovery Rate $(F D R)<0.01$ are ranked by enrichment score $(-\log F D R)$ in Figure $4(a)$. FDR is to conceptualize the rate of type I errors in null hypothesis testing when conducting multiple comparison [70]. Most targets are found to be closely related to several biological processes, such as the steroid-hormone-mediated signaling pathway, inflammatory response, retinoic acid receptor signaling pathway, intracellular receptor signaling pathway, and cellular response to lipopolysaccharide. Moreover, most biological process terms listed have been shown to be highly associated with the immunity, inflammation, and/or eczema. In affected or nonaffected skin of patients with $\mathrm{AD}$, the retinoid transport, synthesis, concentration, and signaling 


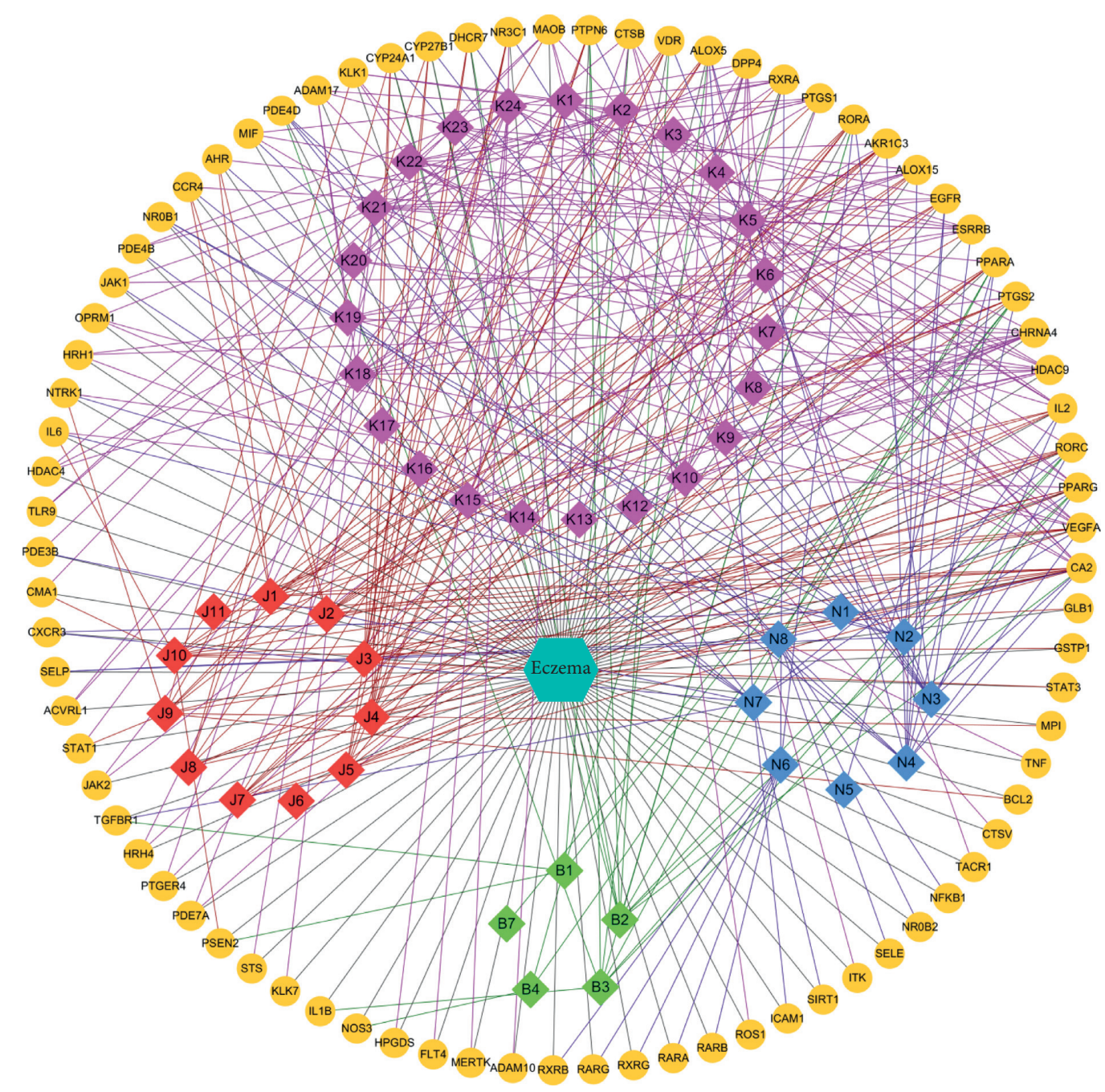

Figure 3: Compound-Target-Disease (C-T-D) network of the SZT formula for eczema treatment. Diamonds, circles, and turquoise hexagon represent bioactive compounds from SZT, corresponding targets associated with eczema, and focused disease eczema. Purple, red, blue, and green diamonds are active compounds from Kushen, Jingiie, Niubangzi, and Baizhu, respectively. Purple, red, blue, and green lines stand for target interactions between Kushen, Jingjie, Niubangzi, and Baizhu, respectively. Black lines indicate relationships between targets and disease eczema. The average degree of networks is 6.3 .

were reported to be strongly decreased, suggesting the intrinsic influence of the retinoid signaling pathway to the pathological process of AD [71]. Particularly, the steroidhormone-mediated signaling pathway with hub targets, such as PPARG, RXRA, RORC, and VDR, is an important regulatory pathway in immunity and inflammation [72]. Inflammatory response, positive regulation of the nitric oxide biosynthetic process, and response to lipopolysaccharide are strongly associated with the inflammatory reaction of the body. The GO results imply that SZT might treat eczema by integral regulating the immune and inflammatory biological processes.

A total of 137 pathways with FDR $<0.01$ were obtained in KOBAS. The top 15 most significant pathways are listed in Figure 4(b). Most pathways are strongly associated with the immune process, metabolic process, and complex pathways in combination of both. For instance, Th17 cell differentiation, inflammatory bowel disease, tuberculosis, kaposisarcoma-associated herpesvirus infection, and human cytomegalovirus infection are more inclined to the immune response induced by internal and/or external stimuli, while the AGE-RAGE signaling pathway in diabetic complications, metabolic pathways, and adipocytokine signaling pathway are more prone to the mediation of the metabolic process in the body. Moreover, both pathways in cancer and the PI3K-Akt signaling pathway are complex and are related to both immune and metabolic processes. Compared with biological process GO analysis, KEGG pathway enrichment provided more detailed information about the signal transmission during biological processes. Thus, key pathways were selected to establish further the C-T-P-D network to determine the relatively critical compounds and hub targets.

A C-T-P-D network was established to reveal the relationships between compounds, targets, pathways and disease.

We speculate that hub targets with high degree in the network are the most promising targets regulated by SZT. 


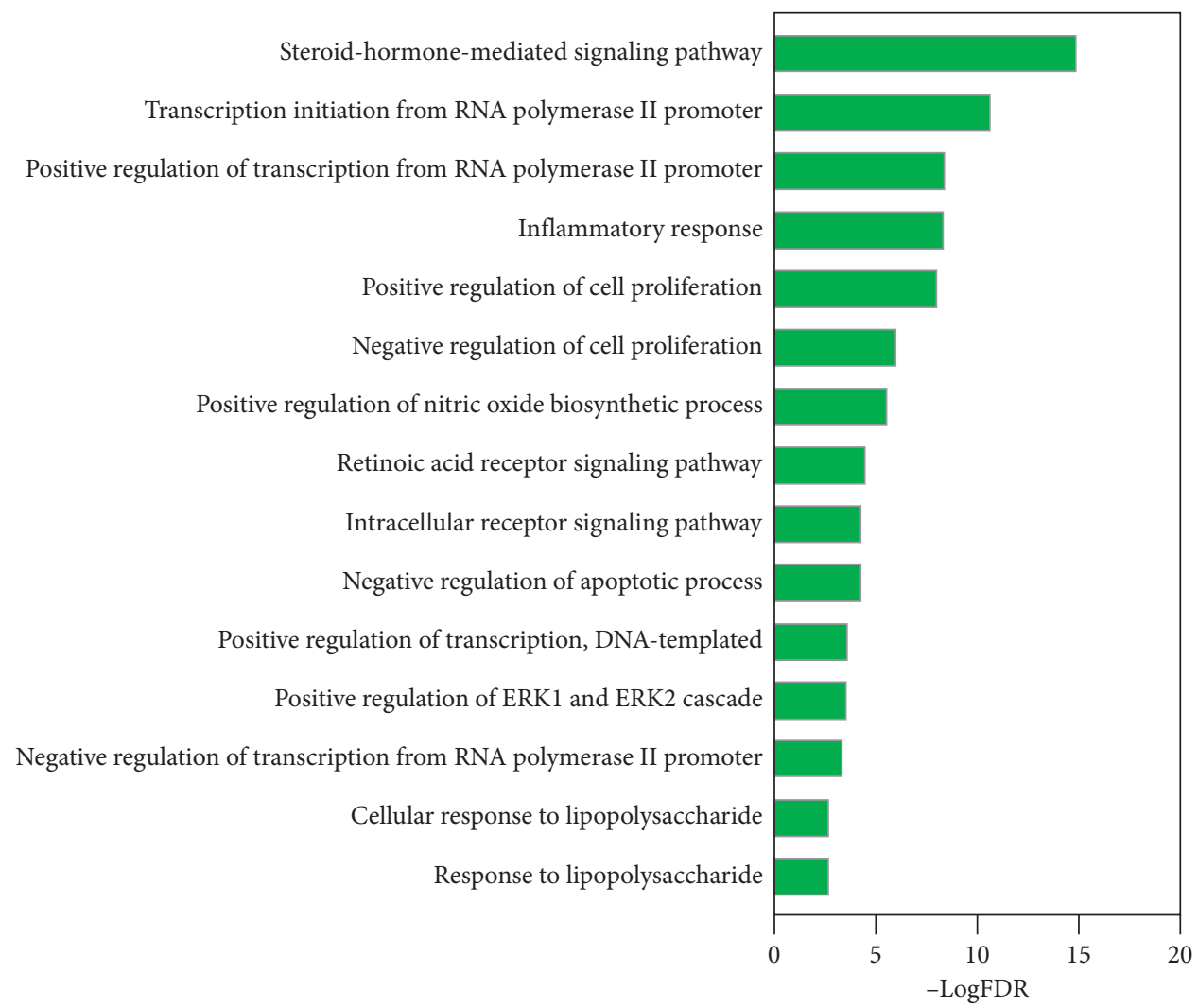

(a)

Th17 cell differentiation

Pathways in cancer

AGE-RAGE signaling pathway in diabetic complications

Parathyroid hormone synthesis, secretion, and action

Inflammatory bowel disease (IBD)

Tuberculosis

Metabolic pathways

PI3K-Akt signaling pathway

Adipocytokine signaling pathway

Kaposi sarcoma-associated herpesvirus infection

Leishmaniasis

Measle
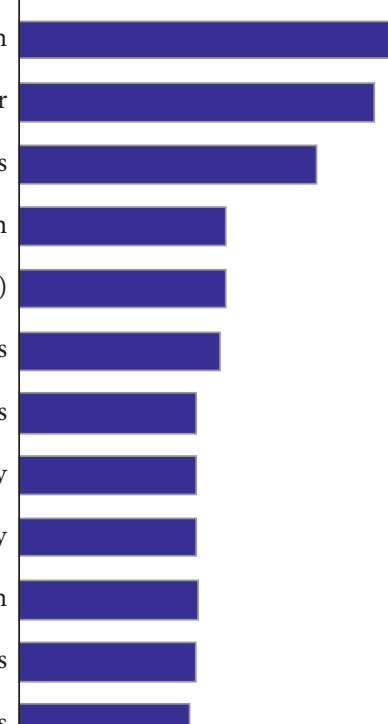

Fluid shear stress and atherosclerosi

PD-L1 expression and PD-1 checkpoint pathway in cancer

Human cytomegalovirus infection

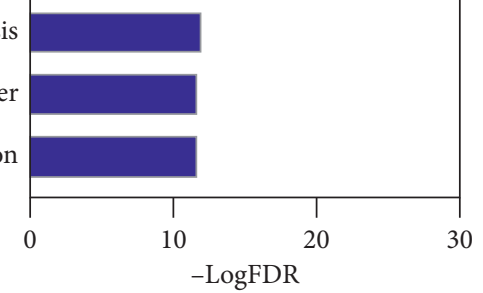

(b)

Figure 4: Gene ontology (GO) and pathway analyses. (a) GO biological process analysis of the targets. $Y$-axis: top 15 biological processes relevant to the enriched targets; $X$-axis: significance of each term ranked with $-\log$ (false discovery rate) (FDR). (b) Pathway analysis of the targets. $Y$-axis: top 15 significant canonical pathways relevant to the enriched targets; $X$-axis: significance of each pathway ranked by $-\log (\mathrm{FDR})$. 
Thus, based on the number of hub targets contained in these pathways, four key pathways were selected, including the Th17 cell differentiation pathway (P1), pathways in cancer (P2), metabolic pathways (P3), and PI3K-Akt signaling pathway (P4). Then, the compounds and targets involved with these four pathways were chosen to construct the C-T-P network, as shown and listed in Figure 5(a). Diamonds, circles, triangles, and hexagons represent bioactive compounds, corresponding targets, pathways, and disease eczema, respectively. Node color from green to red and node size are proportional to its degree in the C-T-P-D network. The network average degree of node was 7.6, with the degree defined as the number of connections. Moreover, detailed information including the involved hub targets, related pathways, and potential ligands is displayed in Figure 5(b). The imbalance of Th1 and Th2 in the pathogenesis of eczema has been widely investigated [73]. Recently, the crucial role of Th17 cell in allergic contact dermatitis has also been demonstrated by several studies. Increasing Th17 activation was confirmed in Asian and pediatric $\mathrm{AD}[74,75]$. Once activated, Th17 cells have the capacity to produce IL17A, IL-17F, IL-22, and IL-26. In particular, IL-17 leads to the expression of proinflammatory cytokines, responsible for eosinophil- and neutrophil-mediated inflammation [76]. We found that 16 targets were involved in the Th17 cell differentiation pathway, including hub targets IL-2, RORC, RORA, and RXRA. Among them, IL-2 is released by activated Th1 cells to regulate inflammation and tissue damage. The regulatory roles of RORs and RXRs in the function of lymphoid cell have already been discussed previously $[60,61]$. Pathways in cancer, including the MAPK signaling pathway, Jak-STAT signaling pathway, calcium signaling pathway, estrogen signaling pathway, and VEGF signaling pathway, were all identified in the pathway analysis. The hub targets, such as ProstaglandinEndoperoxide Synthase 2 (PTGS2), EGFR, RXRA, PPARG, VEGFA, and IL-2, were all included in this complex pathway, pathway in cancer. PTGS2, also known as cyclooxygenase-2 (COX-2), is currently the widely used anti-inflammation target of nonsteroidal anti-inflammatory drugs. The inhibition of PTGS2 results in preferable anti-inflammatory effects [77]. In addition to immune function, the association between $\mathrm{AD}$ and metabolic syndrome has attracted more attention [78]. Metabolic syndrome includes abdominal obesity, hypertension, insulin resistance, and dyslipidemia [79]. Collective data indicate that central obesity is positively associated with $\mathrm{AD}$, which is likely linked to low-grade systemic inflammation induced by adipocytes (leptin and adiponectin) generated by visceral adipose tissue [80]. CA2, VDR, PTGST1, and PTGST2 are all included in metabolic pathways. The PI3K-Akt signaling pathway is involved in a wide variety of cellular processes, including cellular growth, migration, and proliferation, especially the process of inflammation and immune response. It was reported that the expression of PI3K and Akt in blood was significantly higher in AD patients than in the healthy controls, which might be associated with the intrinsic activation of T-cell and cytokine secretion [81]. Moreover, specific inhibition of PI3K expression led to significant inhibition of T-cell proliferation and secretion of cytokines, such as IL-6 and IL-10. The PI3K-Akt signaling pathway has been reported to be associated with LPSinduced acute inflammatory responses and autophagy regulation in immune response [19].

3.4. Molecular Docking Analysis Investigates the Binding Activities. To investigate binding between potential targets and active compounds, in silico molecular docking was used to calculate the binding energy and evaluate the binding mode between the compound and its target. From hub targets, CA2 (P3), PPARG (P2), and RXRA (P1, P2, and $\mathrm{P} 4)$ were selected to represent the four different pathways. Additionally, activation of VDR causes marked induction of skin barrier genes and antimicrobial peptide genes in lesion skin, ameliorating allergen-triggered eczema in the murine AD model [82]. Also, it is a potentially novel therapeutic target for treatment of eczema. Thus, molecular docking was conducted for CA2, PPARG, RXRA, and VDR and their interacting active compounds using AutoDock Vina, considering proteins as rigid molecules. For CA2, promising ligands are docking into receptorbinding-domain as inhibitors. Also, for PPARG, RXRA, and VDR, promising ligands are calculated as their agonists. The ligands and their molecular dockings were demonstrated in Figure 6. The results revealed that all compounds as predicted ligands show moderate to strong binding affinity with related proteins. In particular, compounds cynarine (N8), stigmasterol (J5), (24S)-24-propylcholesta-5-ene-3 $\beta$-ol (B2), kushenol (K1), and $\beta$-sitosterol (N3) had the strongest binding affinities to their receptors. The binding modes of complexes CA2-N8 (-9.1 kcal/mol), PPARG-J5 (-9.3 kcal/mol), PPARG-B2 $(-9.2 \mathrm{kcal} / \mathrm{mol}), \mathrm{RXRA}-\mathrm{K} 1(-8.1 \mathrm{kcal} / \mathrm{mol})$, and VDR-N3 $(-10.6 \mathrm{kcal} / \mathrm{mol})$ are shown in Figure 7.

For CA2, 15 ligands were docked into the CA2 active site and the cynarine (N8) showed highest binding affinity. The hydrophobic pocket formed by Val121, Val143, and Leu198 of CA2 were also observed in the binding of CA2-N8. The hydrogen bond formed between the ligand and Thr199, Thr200, Asn67, and Asp72 further increases the stability of the ligand in the binding site (Figures $7(\mathrm{a})$ and $7(\mathrm{~b})$ ). For PPARG, both stigmasterol (J5) and (24S)-24-propylcholesta-5-ene-3 $\beta$-ol (B2) were well fitted into the binding cavity of the protein by hydrophobic interaction. The binding pocket of PPARG-stigmasterol (J5) shown in Figures 7(c) and $7(\mathrm{~d})$ indicates that nine amino acid residues from PPARG interacted with J5 by hydrophobic interactions, including Leu330, Ile326, Val339, Arg288, Cyc285, Ile341, Leu270, and Ile262. Both stigmasterol and (24S)-24-propylcholesta-5-ene-3 $\beta$-ol are phytosterols with similar chemical structure. The binding energy of PPARG-B2 (Figures $7(\mathrm{e})$ and $7(\mathrm{f})$ ) is $-9.2 \mathrm{kcal} / \mathrm{mol}$, very close to that of PPARG-stigmasterol (J5) $(-9.3 \mathrm{kcal} / \mathrm{mol})$. Moreover, the binding of PPARG-N3 ( $\beta$-sitosterol) has previously been studied [83] with similar affinity. For RXRA, docking ligands 


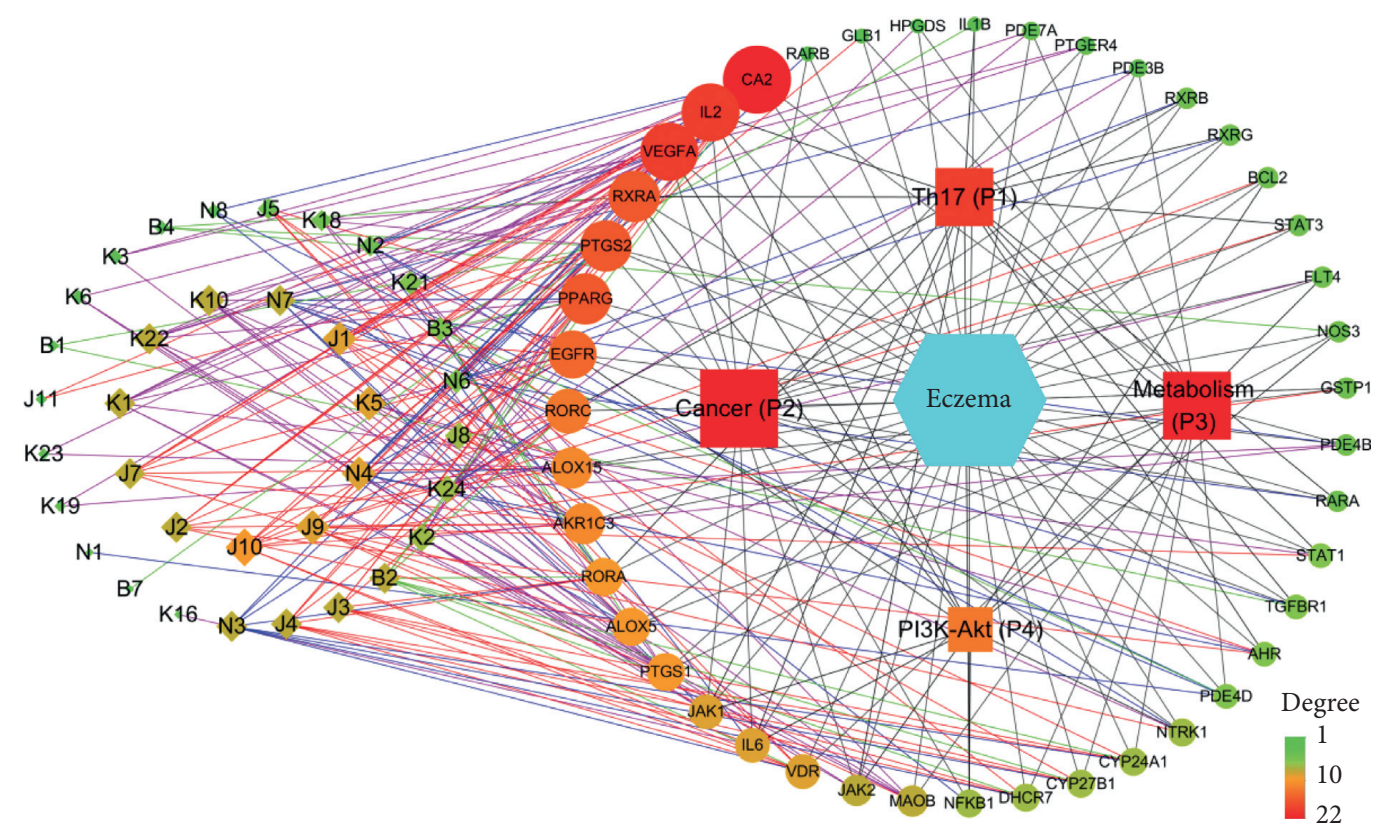

(a)

\begin{tabular}{|l|c|c|c|}
\hline Pathways & Hub targets & Degree & \multicolumn{1}{|c|}{ Related compounds } \\
\hline P3 & CA2 & 16 & K1, 3, 5, 21, 22; N3, 4, 8; J1, 2, 4, 7, 8, 9, 11; B2. \\
\hline P2, 4 & VEGFA & 12 & K1, 5, 18, 19, 22, 23; N2, 4; J1, 2, 7, 10. \\
\hline P2 & PPARG & 11 & K1, 2, 10, 22; N3, N7; J4, 5, 9; B2, 3. \\
\hline P1, 2, 4 & IL2 & 10 & K1, 3, 5, 21; N2, 4; J1, 2, 7, 8. \\
\hline P2, 3 & PTGS2 & 10 & K5, 18; N4; J1, 7, 10, 11; B1, 4, 7. \\
\hline P1, 2, 4 & RXRA & 8 & K1, 2, 10, 18, 22; N6; J9; B4. \\
\hline P1 & RORC & 9 & K24; N3, N7; J4, 5, 8, 10; B2, 3. \\
\hline P1 & RORA & 7 & N3, 6; J4, 5, 8; B2, 3 \\
\hline P2, 4 & EGFR & 9 & K5, 21, 24; N2, 4; J1, 2, 7, 10. \\
\hline P3 & PTGS1 & 8 & K1, 2, 3, 10, 18, 21, 22; J9. \\
\hline P3 & VDR & 6 & N3; J4, 5, 8, 10; B2. \\
\hline
\end{tabular}

(b)

Figure 5: Compound-Target-Pathway-Disease (C-T-P-D) network of the SZT formula for eczema treatment. (a) C-T-P-D network. Square block: the important pathway regulated by SZT; diamonds and circles: bioactive compounds and corresponding targets associated with the four pathways. Turquoise hexagon: disease eczema. Purple, red, blue, and green lines: target interactions between Kushen, Jingjie, Niubangzi, and Baizhu, respectively. Black line: the interaction between targets and the related pathways; the relationship between targets and disease eczema. Node color from green to red and node size are proportional to its degree. (b) Relationship table for pathways, hub targets, and compounds.

into the ligand-binding domain was used to evaluate the possibility of these compounds acting as agonists. The strongest binding existed between RXRA and kushenol (K1). Hydrogen binding (Cys432), $\pi-\pi$ stacking (Phe313), and hydrophobic interactions (Ile268, Ala271, Ala272, and Leu309) formed between protein residues and ligand kushenol (K1) were illustrated in Figures 7(g) and 7(h). VDR is a member of nuclear receptor superfamily of transcriptional regulators, which can be activated by its specific ligand vitamin $\mathrm{D}_{3}$. The docking result indicated that binding conformation of $\beta$-sitosterol (N3) fitted well in binding domain of VDR crystallographical structure forming stable complex VDR-N3 ( $\beta$-sitosterol). All 14 amino acid residues showed hydrophobic interactions with ligand N3 ( $\beta$-sitosterol) (Figures 7(i) and 7(j)). Their forceful interaction might attribute to the similar structure of vitamin $\mathrm{D}_{3}$ and $\beta$-sitosterol. Inspiringly, it has been reported that $\beta$-sitosterol promoted the immune function of vitamin $\mathrm{D}_{3}$ [84], suggesting the anti-inflammation and immune enhancement function of $\beta$-sitosterol. The abovementioned strong 


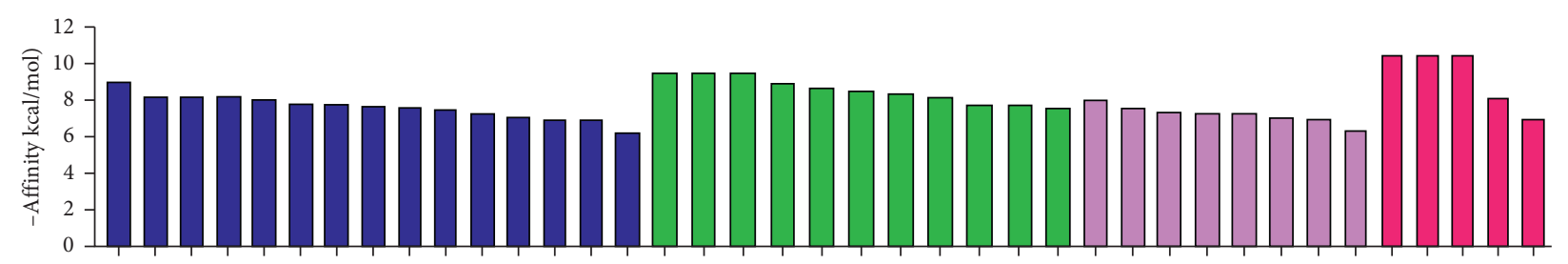

Compounds: N8 K3 J8 K21N4 J2 J7 N3 K1 K22 K5 B2 J9 J11 J4 J5
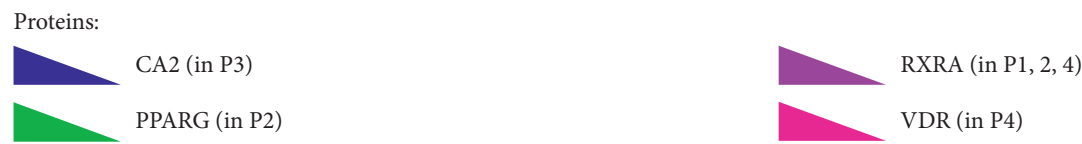

Figure 6: Molecular docking results between proteins and promising ligands. Blue bars: docking affinity between CA2 and 15 potential ligands; green: PPARG and 11 potential ligands; purple: RXRA and 8 potential ligands; and red: VDR and 5 potential ligands.

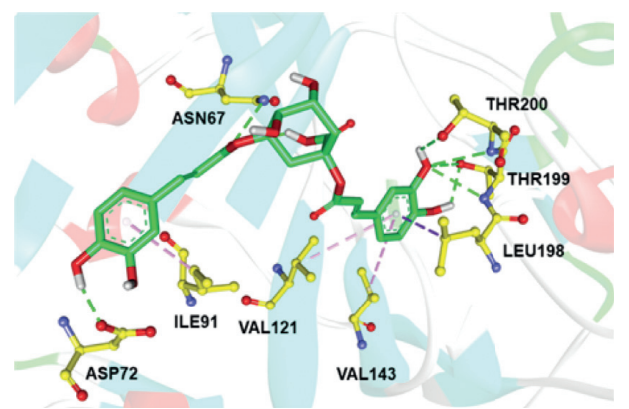

(a)

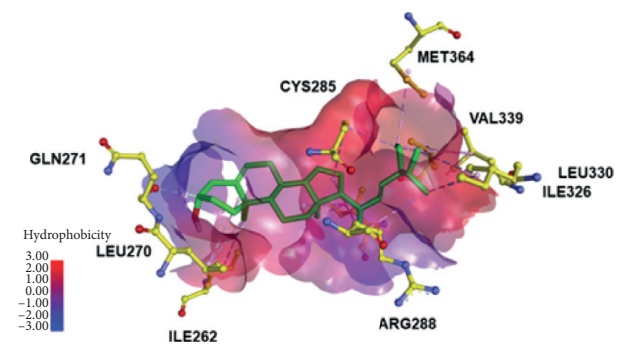

(c)

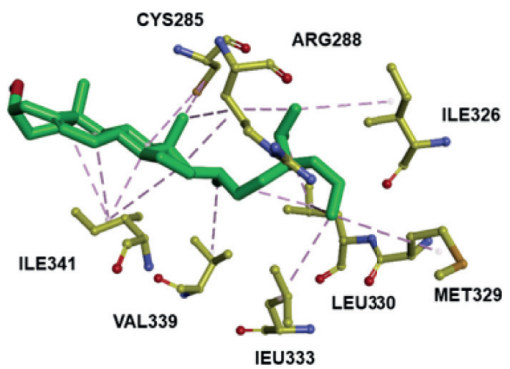

(e)

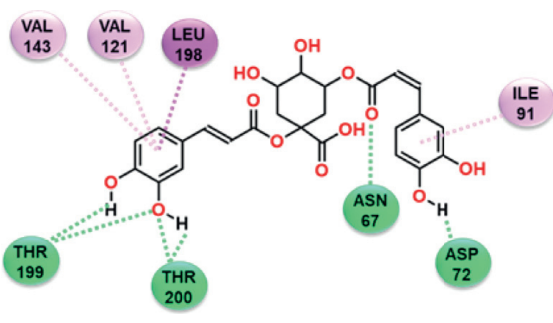

(b)

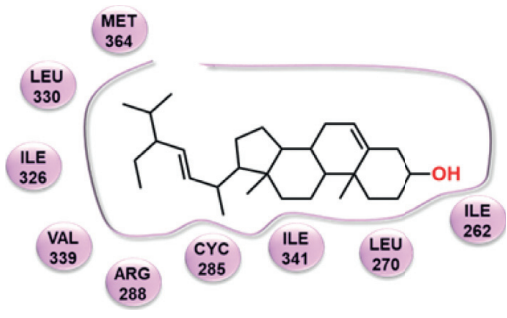

(d)

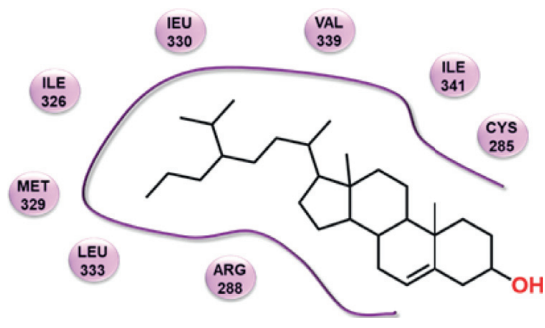

(f)

FIgUre 7: Continued. 


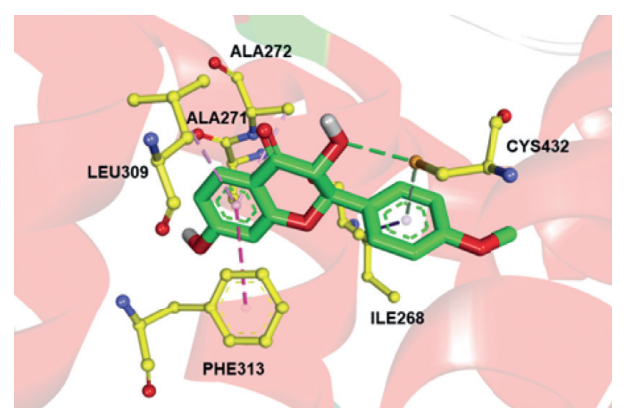

(g)

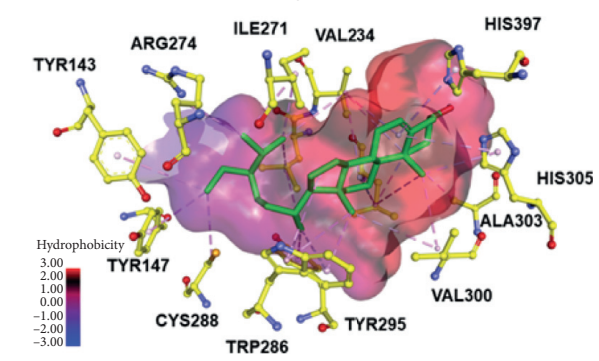

(i)

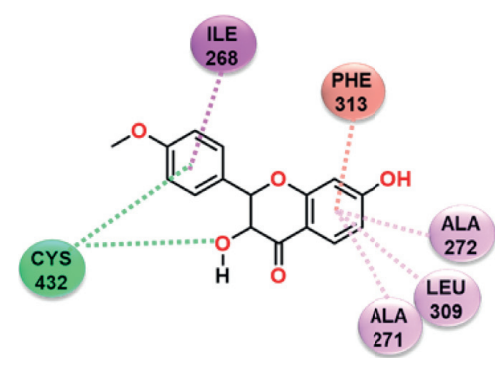

(h)

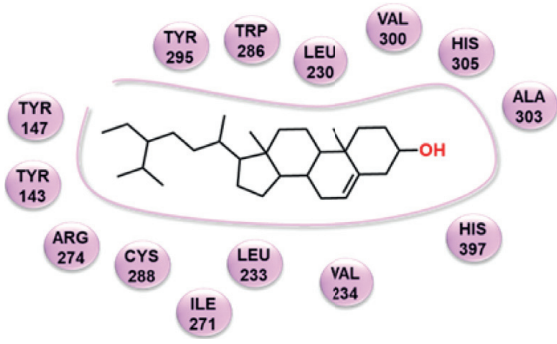

$(\mathrm{j})$

Figure 7: Binding explorations of complex CA2-N8, PPARG-J5, PPARG-B2, RXRA-K1, and VDR-N3. (a) Predicted binding mode of CA2 (PDB: 2Q38) with lowest-energy docking ligand N8 in the active pocket. (b) Two-dimensional (2D) binding view of N8 with CA2. (c) Hydrophobic discrepancy of compound J5 with PPARG (PDB: 2PRG) in the active pocket. (d) 2D binding view of J5 with PPARG. (e) Hydrophobic discrepancy of compound B2 with PPARG in the active pocket. (f) 2D binding view of B2 with PPARG. (g) Predicted binding mode of RXRA with $\mathrm{K} 1$ in the active pocket (PDB: 1MVC). (h) 2D binding view of K1 with RXRA. (i) Hydrophobic discrepancy of compound N3 with VDR (PDB: 3A3Z) in the active pocket. (j) 2D binding view of N3 with VDR. Left panel: the carbon and oxygen of ligands are highlighted in green and red, respectively. The yellow, red, and blue binding residues stand for $\mathrm{C}, \mathrm{O}$, and $\mathrm{N}$, respectively. Right panel: the green, pink, and purple stand for hydrogen binding, $\pi-\pi$ stacking, andhydrophobic interaction between ligand and related residents, respectively.

interactions further imply the functioning route of SZT compounds through proteins and their corresponding biological activities.

\section{Conclusions}

Eczema is a chronic inflammatory condition causing pruritus, erythema, dryness, scaling, and vesiculopapular rash that negatively impact the quality of life of the affected patients [1]. With no definite cure for eczema, common medicines, such as oral antihistamines, topical corticosteroids, emollients, and even immune-modulating agents, can not only relieve symptoms but also sometimes cause severe side effects [85]. Significantly, these agents do not target upstream molecular dysregulation that is central to eczema. There is an urgent need to develop new treatments to not only relieve symptoms but also address the underlying molecular mechanisms that drive the disease to potentially cure eczema with fewer side effects. TCM has a long and well-known history in Asia treating diseases in a multicompound-multitarget modality [86]. TCM as a complementary or alternative medicine is gradually becoming more widely accepted throughout the world, and molecular basis of its activity is only recently being elucidated [87]. XFS is an acclaimed TCM formula in China documented in "Orthodox Manual of External Medicine" [13], which eliminates itching in the body. We modified the formula to retain four pivotal herbs to develop SZT that has excellent efficacy in treatment of eczema. However, molecular mechanisms of SZT remain poorly defined. Recently, systems pharmacology based on network analysis has provided a new method to uncover the mechanisms of complex formula in TCM [16-18]. We explored the molecular mechanisms of SZT for eczema treatment using a systems pharmacology approach, including ADME evaluation, herb feature mapping, drug target mining, network and pathway analyses, and in silico molecular docking.

In summary, we found 51 active compounds out of 441 compounds that were retrieved based on $\mathrm{OB}$ and $\mathrm{DL}$ screening with satisfactory pharmacokinetic properties. We further studied these active compounds for their physicochemical properties and drug-likeness. Findings of drug target mining supported that active compounds targeted single or multiple proteins involved in the pathological process or treatment of eczema. The C-T-D network analysis, GO, and pathway analyses conducted using DAVAD and KOBAS databases allowed us to elucidate the pharmacological effects of SZT from a holistic perspective. Our results imply that SZT efficacy in eczema may be attributed to immune and metabolic functions via regulation of multiple pathways and networks. Among these, four key pathways stood out, including Th17 cell differentiation, pathways in cancer, metabolic pathways, and PI3K-Akt 
signaling pathway. Moreover, the C-T-P-D network highlighted the critical active compounds. Finally, molecular docking studies between active compounds and proteins CA2, PPARG, RXRA, and VDR confirmed moderate to strong binding affinities. These data further support the potential biological activities of these active compounds. Overall, the formula affects a regulation of the immune system and metabolic processes, instead of a single target or a single pathway. In vitro and in vivo studies are currently in progress to investigate, confirm, and expand knowledge into the molecular alterations induced by SZT active compounds. In conclusion, systems pharmacology was applied in this work to shed light on the molecular mechanism and pharmacological regulation of SZT for eczema treatment. Our results will help further optimize SZT and facilitate development of more effective drugs to cure eczema.

\section{Data Availability}

The data used to support the findings of this study are included within the article.

\section{Conflicts of Interest}

ZZW, YL, WH, RT, ANW, JG, and MM have no financial conflicts of interest to disclose. KDS receives salary from General Nutraceutical Technology LLC. XML received research support from the National Institutes of Health (NIH)/ National Center for Complementary and Alternative Medicine (NCCAM), Food Allergy Research and Education (FARE) and Winston Wolkoff Integrative Medicine Fund for Allergies and Wellness, the Parker Foundation, and Henan University of Chinese Medicine; received consulting fees from Food Allergy Research and Education (FARE), Johnson \& Johnson Pharmaceutical Research \& Development, LLC, and Bayer Global Health LLC; royalties from UpToDate; travel expenses from the National Center for Complementary and Alternative Medicine (NCCAM) and FARE, Henan University of Chinese Medicine, Harvard TCM summit, China Allergy Society Conference, and North American TCM Symposium; shares US patents PCT/US05/ 008417, PCT/US 10,500,169, PCP/14/762,416, and PCP 14/ 762,416 ; is a member of Herbs Springs, LLC, General Nutraceutical Technology LLC, and Health Freedom LLC; and takes compensation from practice at Integrative Health and Acupuncture PC.

\section{Acknowledgments}

This research was funded by the Henan University of Chinese Medicine, visiting fellowship grant to ZZW (CTIM01-2019), and Scientific Research Foundation (KYQD 201905). This research was also supported by the Henan Province Scientific and Technological Project (no. 202102310472) and Study of Integrative Medicine forImmunology and Wellness, New York Medical College. The authors thank Henry Ehrlich for critical reading of the manuscript; Wei Zhou,
Lihua Cao and Hongjuan He, Nan Yang, Anish R Maskey, $\mathrm{Ke} \mathrm{Li}$, and Ibrahim A Musa for helpful discussion.

\section{References}

[1] N. Novak, "New insights into the mechanism and management of allergic diseases: atopic dermatitis," Allergy, vol. 64, no. 2, pp. 265-275, 2009.

[2] T. E. Shaw, G. P. Currie, C. W. Koudelka, and E. L. Simpson, "Eczema prevalence in the United States: data from the 2003 national survey of children's health," Journal of Investigative Dermatology, vol. 131, no. 1, pp. 67-73, 2011.

[3] J. I. Silverberg, "Health care utilization, patient costs, and access to care in US adults with eczema," JAMA Dermatology, vol. 151, no. 7, pp. 743-752, 2015.

[4] P. D. Arkwright, C. Motala, H. Subramanian, J. Spergel, L. C. Schneider, and A. Wollenberg, "Management of difficult-to-treat atopic dermatitis," The Journal of Allergy and Clinical Immunology: In Practice, vol. 1, no. 2, pp. 142-151, 2013.

[5] R. J. Hay, N. E. Johns, H. C. Williams et al., "The global burden of skin disease in 2010: an analysis of the prevalence and impact of skin conditions," Journal of Investigative Dermatology, vol. 134, no. 6, pp. 1527-1534, 2014.

[6] C. J. Apfelbacher, A. Jupiter, B. Carter, E. Weisshaar, and M. M. Böhmer, “Oral H1 antihistamines as 'add-on' therapy to topical treatment for eczema," Cochrane Database of Systematic Reviews, vol. 4, 2016.

[7] R. Nowicki, M. Trzeciak, A. Wilkowska et al., "Special paper atopic dermatitis: current treatment guidelines. Statement of the experts of the dermatological section, polish society of allergology, and the allergology section, polish society of dermatology," Advances in Dermatology and Allergology, vol. 4, no. 4, pp. 239-249, 2015.

[8] X.-M. Li and H. Ehrlich, Traditional Chinese Medicine, Western Science, and the Fight against Allergic Disease, World Scientific Publishing, Singapore, 1st edition, 2016.

[9] K. L. E. Hon, T. F. Leung, P. C. Ng et al., "Efficacy and tolerability of a Chinese herbal medicine concoction for treatment of atopic dermatitis: a randomized, double-blind, placebo-controlled study," British Journal of Dermatology, vol. 157, no. 2, pp. 357-363, 2007.

[10] S. Gu, A. W. H. Yang, C. C. L. Xue et al., "Chinese herbal medicine for atopic eczema," Cochrane Database of Systematic Reviews, vol. 9, 2013.

[11] X.-R. Lin, C.-X. Tu, X.-M. Meng, C.-M. Yang, M.-Y. Gao, and L. Gu, "Studies on treating eczema by Chinese herbal medicine with anti-type IV allergic activity," Chinese Journal of Integrated Traditional and Western Medicine, vol. 7, no. 1, pp. 7-11, 2001.

[12] D. Huang, K. Chen, F.-R. Zhang et al., "Efficacy and safety of Run Zao Zhi Yang capsule on chronic eczema: a multiplecenter, randomized, double-blind, placebo-controlled clinical study," Journal of Dermatological Treatment, vol. 30, no. 7, pp. 677-684, 2019.

[13] Y.-H. Lin, Y.-C. Chen, S. Hu, H.-Y. Chen, J.-L. Chen, and S.-H. Yang, "Identifying core herbal treatments for urticaria using Taiwan's nationwide prescription database," Journal of Ethnopharmacology, vol. 148, no. 2, pp. 556-562, 2013.

[14] H.-Y. Chen, Y.-H. Lin, S. Hu, S.-h. Yang, J.-l. Chen, and Y.-C. Chen, "Identifying Chinese herbal medicine network for eczema: implications from a nationwide prescription database," Evidence-Based Complementary and Alternative Medicine, vol. 2015, Article ID 347164, 9 pages, 2015. 
[15] E. Thanik, J. A. Wisniewski, A. Nowak-Wegrzyn, H. Sampson, and X.-M. Li, "Effect of traditional Chinese medicine on skin lesions and quality of life in patients with moderate to severe eczema," Annals of Allergy, Asthma \& Immunology, vol. 121, no. 1, pp. 135-136, 2018.

[16] J. Hansen and R. Iyengar, "Computation as the mechanistic bridge between precision medicine and systems therapeutics," Clinical Pharmacology \& Therapeutics, vol. 93, no. 1, pp. 117-128, 2013.

[17] S. Zhao and R. Iyengar, "Systems pharmacology: network analysis to identify multiscale mechanisms of drug action," Annual Review of Pharmacology and Toxicology, vol. 52, no. 1, pp. 505-521, 2012.

[18] M. Danhof, "Systems pharmacology—towards the modeling of network interactions," European Journal of Pharmaceutical Sciences, vol. 94, pp. 4-14, 2016.

[19] M. Zhao, Y. Chen, C. Wang et al., "Systems pharmacology dissection of multi-scale mechanisms of action of Huo-XiangZheng-Qi formula for the treatment of gastrointestinal diseases," Frontiers in Pharmacology, vol. 9, p. 1448, 2019.

[20] X. Li, X. Xu, J. Wang et al., "A system-level investigation into the mechanisms of Chinese Traditional Medicine: compound Danshen Formula for cardiovascular disease treatment," PLoS One, vol. 7, no. 9, Article ID e43918, 2012.

[21] Y. Liu, H. Xing, X. Jiang, Y. Chen, M. Huang, and S. Yu, "Network pharmacology-based preventive effect of XZF on cutaneous toxicities induced by EGFR inhibitor," Biomedicine \& Pharmacotherapy, vol. 123, Article ID 109755, 2020.

[22] J. Li, P. Zhao, Y. Li, Y. Tian, and Y. Wang, "Systems pharmacology-based dissection of mechanisms of Chinese medicinal formula Bufei Yishen as an effective treatment for chronic obstructive pulmonary disease," Scientific Reports, vol. 5, no. 1, Article ID 15290, 2015.

[23] W. Zhou, Z. Chen, W. Li et al., "Systems pharmacology uncovers the mechanisms of anti-asthma herbal medicine intervention (ASHMI) for the prevention of asthma," Journal of Functional Foods, vol. 52, pp. 611-619, 2019.

[24] J. Ru, P. Li, J. Wang et al., "TCMSP: a database of systems pharmacology for drug discovery from herbal medicines," Journal of Cheminformatics, vol. 6, no. 1, p. 13, 2014.

[25] Y. Yamanishi, M. Kotera, M. Kanehisa, and S. Goto, "Drugtarget interaction prediction from chemical, genomic and pharmacological data in an integrated framework," Bioinformatics, vol. 26, no. 12, pp. i246-i254, 2010.

[26] H. Liu, J. Wang, W. Zhou, Y. Wang, and L. Yang, "Systems approaches and polypharmacology for drug discovery from herbal medicines: an example using licorice," Journal of Ethnopharmacology, vol. 146, no. 3, pp. 773-793, 2013.

[27] C. A. Lipinski, F. Lombardo, B. W. Dominy, and P. J. Feeney, "Experimental and computational approaches to estimate solubility and permeability in drug discovery and development settings," Advanced Drug Delivery Reviews, vol. 23, no. $1-3$, pp. 3-25, 1997.

[28] A. Daina, O. Michielin, and V. Zoete, "SwissTargetPrediction: updated data and new features for efficient prediction of protein targets of small molecules," Nucleic Acids Research, vol. 47, no. W1, pp. W357-W364, 2019.

[29] M. J. Keiser, B. L. Roth, B. N. Armbruster, P. Ernsberger, J. J. Irwin, and B. K. Shoichet, "Relating protein pharmacology by ligand chemistry," Nature Biotechnology, vol. 25, no. 2, pp. 197-206, 2007.

[30] S. Kim, J. Chen, T. Cheng et al., "PubChem 2019 update: improved access to chemical data," Nucleic Acids Research, vol. 47, no. D1, pp. D1102-D1109, 2018.
[31] D. S. Wishart, C. Knox, A. C. Guo et al., "DrugBank: a knowledgebase for drugs, drug actions and drug targets," Nucleic Acids Research, vol. 36, no. suppl_1, pp. D901-D906, 2007.

[32] Y. Wang, S. Zhang, F. Li et al., "Therapeutic target database 2020: enriched resource for facilitating research and early development of targeted therapeutics," Nucleic Acids Research, vol. 48, no. D1, pp. D1031-D1041, 2019.

[33] J. Piñero, J. M. Ramírez-Anguita, J. Saüch-Pitarch et al., "The DisGeNET knowledge platform for disease genomics: 2019 update," Nucleic Acids Research, vol. 48, no. D1, pp. D845D855, 2019.

[34] D. Carvalho-Silva, A. Pierleoni, M. Pignatelli et al., "Open targets platform: new developments and updates two years on," Nucleic Acids Research, vol. 47, no. D1, pp. D1056-D1065, 2018.

[35] T. U. Consortium, "UniProt: a worldwide hub of protein knowledge," Nucleic Acids Research, vol. 47, no. D1, pp. D506-D515, 2018.

[36] D. W. Huang, B. T. Sherman, and R. A. Lempicki, "Bioinformatics enrichment tools: paths toward the comprehensive functional analysis of large gene lists," Nucleic Acids Research, vol. 37, no. 1, pp. 1-13, 2008.

[37] C. Xie, X. Mao, J. Huang et al., "Kobas 2.0: a web server for annotation and identification of enriched pathways and diseases," Nucleic Acids Research, vol. 39, no. suppl_2, pp. W316-W322, 2011.

[38] Y. Assenov, F. Ramírez, S.-E. Schelhorn, T. Lengauer, and M. Albrecht, "Computing topological parameters of biological networks," Bioinformatics, vol. 24, no. 2, pp. 282-284, 2007.

[39] O. Trott and A. J. Olson, "AutoDock Vina: improving the speed and accuracy of docking with a new scoring function, efficient optimization, and multithreading," Journal of Computational Chemistry, vol. 31, no. 2, pp. 455-461, 2010.

[40] H. M. Berman, J. Westbrook, Z. Feng et al., "The protein data bank," Nucleic Acids Research, vol. 28, no. 1, pp. 235-242, 2000.

[41] M. F. Sanner, "Python: a programming language for software integration and development," Journal of Molecular Graphics \& Modelling, vol. 17, no. 1, pp. 57-61, 1999.

[42] W. L. DeLano, "Pymol: an open-source molecular graphics tool," CCP4 Newsletter on Protein Crystallography, vol. 40, pp. 82-92, 2002.

[43] DassaultSystèmesBIOVIAD, “Discovery studio,” 2020.

[44] D. F. Veber, S. R. Johnson, H.-Y. Cheng, B. R. Smith, K. W. Ward, and K. D. Kopple, "Molecular properties that influence the oral bioavailability of drug candidates," Journal of Medicinal Chemistry, vol. 45, no. 12, pp. 2615-2623, 2002.

[45] J. R. Guzman, J. S. Koo, J. R. Goldsmith, M. Mühlbauer, A. Narula, and C. Jobin, "Oxymatrine prevents NF- $\kappa$ B nuclear translocation and ameliorates acute intestinal inflammation," Scientific Reports, vol. 3, no. 1, p. 1629, 2013.

[46] Y. Yang, J. Xiu, X. Zhang et al., "Antiviral effect of matrine against human enterovirus 71," Molecules, vol. 17, no. 9, pp. 10370-10376, 2012.

[47] X.-M. Li and L. Brown, "Efficacy and mechanisms of action of traditional Chinese medicines for treating asthma and allergy," Journal of Allergy and Clinical Immunology, vol. 123, no. 2, pp. 297-306, 2009.

[48] H.-M. Oh, S. W. Lee, M. H. Park et al., "Norkurarinol inhibits toll-like receptor 3 (TLR3)-mediated pro-inflammatory signaling pathway and rotavirus replication," Journal of Pharmacological Sciences, vol. 118, no. 2, pp. 161-170, 2012. 
[49] C. M. Park and Y.-S. Song, "Luteolin and luteolin-7-O-glucoside inhibit lipopolysaccharide-induced inflammatory responses through modulation of NF- $\kappa \mathrm{B} / \mathrm{AP}-1 / \mathrm{PI} 3 \mathrm{~K}-\mathrm{Akt}$ signaling cascades in RAW 264.7 cells," Nutrition Research and Practice, vol. 7, no. 6, pp. 423-429, 2013.

[50] J. Mlcek, T. Jurikova, S. Skrovankova, and J. Sochor, "Quercetin and its anti-allergic immune response," Molecules, vol. 21 , no. 5 , p. $623,2016$.

[51] S.-J. Kim, "The ameliorative effect of $\beta$-sitosterol on DNCBinduced atopic dermatitis in mice," Biomedical Science Letters, vol. 23, no. 4, pp. 303-309, 2017.

[52] Q. Gao, M. Yang, and Z. Zuo, "Overview of the anti-inflammatory effects, pharmacokinetic properties and clinical efficacies of arctigenin and arctiin from Arctium lappa L." Acta Pharmacologica Sinica, vol. 39, no. 5, pp. 787-801, 2018.

[53] M. Topal, H. Gocer, F. Topal et al., "Antioxidant, antiradical, and anticholinergic properties of cynarin purified from the Illyrian thistle (Onopordum illyricum L.)," Journal of Enzyme Inhibition and Medicinal Chemistry, vol. 31, no. 2, pp. 266275, 2016.

[54] S. A. Holanda Pinto, L. M. S. Pinto, G. M. A. Cunha, M. H. Chaves, F. A. Santos, and V. S. Rao, "Anti-inflammatory effect of $\alpha, \beta$-Amyrin, a pentacyclic triterpene from Protium heptaphyllum in rat model of acute periodontitis," Inflammopharmacology, vol. 16, no. 1, pp. 48-52, 2008.

[55] F. A. Oliveira, R. C. P. Lima-Junior, W. M. Cordeiro et al., "Pentacyclic triterpenoids, $\alpha, \beta$-amyrins, suppress the scratching behavior in a mouse model of pruritus," Pharmacology Biochemistry and Behavior, vol. 78, no. 4, pp. 719725, 2004.

[56] A. Genovese, A. Detoraki, F. Granata, M. R. Galdiero, G. Spadaro, and G. Marone, "Angiogenesis, lymphangiogenesis and atopic dermatitis," New Trends in Allergy and Atopic Eczema, vol. 96, pp. 50-60, 2012.

[57] A. Sääf, A. Pivarcsi, M. C. G. Winge et al., "Characterization of EGFR and ErbB2 expression in atopic dermatitis patients," Archives of Dermatological Research, vol. 304, no. 10, pp. 773-780, 2012.

[58] K.-H. Hsieh, C.-C. Chou, and S.-F. Huang, "Interleukin 2 therapy in severe atopic dermatitis," Journal of Clinical Immunology, vol. 11, no. 1, pp. 22-28, 1991.

[59] S. Ramalingam, M. Packirisamy, M. Karuppiah et al., "Effect of $\beta$-sitosterol on glucose homeostasis by sensitization of insulin resistance via enhanced protein expression of PPR $\gamma$ and glucose transporter 4 in high fat diet and streptozotocininduced diabetic rats," Cytotechnology, vol. 72, no. 3, pp. 357-366, 2020.

[60] J. Dai, M.-K. Choo, J. M. Park, and D. E. Fisher, “Topical ROR inverse agonists suppress inflammation in mouse models of atopic dermatitis and acute irritant dermatitis," Journal of Investigative Dermatology, vol. 137, no. 12, pp. 2523-2531, 2017.

[61] M. I. Dawson and Z. Xia, "The retinoid X receptors and their ligands," Biochimica et Biophysica Acta (BBA)-Molecular and Cell Biology of Lipids, vol. 1821, no. 1, pp. 21-56, 2012.

[62] J. Mihály, J. Gericke, G. Aydemir et al., "Reduced retinoid signaling in the skin after systemic retinoid-X receptor ligand treatment in mice with potential relevance for skin disorders," Dermatology, vol. 225, no. 4, pp. 304-311, 2012.

[63] N. Malhotra, J. M. Leyva-Castillo, U. Jadhav et al., "ROR $\alpha$ expressing $\mathrm{T}$ regulatory cells restrain allergic skin inflammation," Science Immunology, vol. 3, no. 21, Article ID eaao6923, 2018.
[64] M. Mboge, B. Mahon, R. McKenna, and S. Frost, "Carbonic anhydrases: role in $\mathrm{pH}$ control and cancer," Metabolites, vol. 8, no. 1, p. 19, 2018.

[65] C. T. Supuran, A. Scozzafava, and A. Casini, "Carbonic anhydrase inhibitors," Medicinal Research Reviews, vol. 23, no. 2, pp. 146-189, 2003.

[66] M. Kamsteeg, P. L. J. M. Zeeuwen, G. J. de Jongh et al., "Increased expression of carbonic anhydrase II (CA II) in lesional skin of atopic dermatitis: regulation by Th2 cytokines," Journal of Investigative Dermatology, vol. 127, no. 7, pp. 1786-1789, 2007.

[67] J.-P. Hachem, D. Crumrine, J. Fluhr, B. E. Brown, K. R. Feingold, and P. M. Elias, "pH directly regulates epidermal permeability barrier homeostasis, and stratum corneum integrity/cohesion," Journal of Investigative Dermatology, vol. 121, no. 2, pp. 345-353, 2003.

[68] J. Schalkwijk, M. Kamsteeg, G. J. D. Jongh, and P. L. J. M. Zeeuwen, New Use of Carbonic Anhydrase Inhibitors, University of Saskatchewan, Nijmegen, Netherlands, 2007.

[69] P. Sertznig and J. Reichrath, "Peroxisome proliferator-activated receptors (PPARs) in dermatology," Dermato-Endocrinology, vol. 3, no. 3, pp. 130-135, 2011.

[70] Y. Benjamini and Y. Hochberg, "Controlling the false discovery rate: a practical and powerful approach to multiple testing," Journal of the Royal Statistical Society: Series B (Methodological), vol. 57, no. 1, pp. 289-300, 1995.

[71] J. Mihály, A. Gamlieli, M. Worm, and R. Rühl, "Decreased retinoid concentration and retinoid signalling pathways in human atopic dermatitis," Experimental Dermatology, vol. 20, no. 4, pp. 326-330, 2011.

[72] L. I. McKay and J. A. Cidlowski, "Molecular control of immune/inflammatory responses: interactions between nuclear factor- $\kappa \mathrm{b}$ and steroid receptor-signaling pathways," Endocrine Reviews, vol. 20, no. 4, pp. 435-459, 1999.

[73] K. Eyerich and N. Novak, "Immunology of atopic eczema: overcoming the Th1/Th2 paradigm," Allergy, vol. 68 , no. 8 , pp. 974-982, 2013.

[74] S. Noda, M. Suárez-Fariñas, B. Ungar et al., "The Asian atopic dermatitis phenotype combines features of atopic dermatitis and psoriasis with increased TH17 polarization," Journal of Allergy and Clinical Immunology, vol. 136, no. 5, pp. 12541264, 2015.

[75] P. M. Brunner, A. Israel, N. Zhang et al., "Early-onset pediatric atopic dermatitis is characterized by TH2/TH17/ TH22-centered inflammation and lipid alterations," Journal of Allergy and Clinical Immunology, vol. 141, no. 6, pp. 20942106, 2018.

[76] P. Rerknimitr, A. Otsuka, C. Nakashima, and K. Kabashima, "The etiopathogenesis of atopic dermatitis: barrier disruption, immunological derangement, and pruritus," Inflammation and Regeneration, vol. 37, no. 1, p. 14, 2017.

[77] C. S. Williams, M. Mann, and R. N. DuBois, "The role of cyclooxygenases in inflammation, cancer, and development," Oncogene, vol. 18, no. 55, pp. 7908-7916, 1999.

[78] J. Lee, H. Jung, K. Han et al., "Association between metabolic syndrome and atopic dermatitis in Korean adults," Acta Dermato Venereologica, vol. 97, no. 1, pp. 77-80, 2017.

[79] E. Reisin and M. A. Alpert, "Definition of the metabolic syndrome: current proposals and controversies," The American Journal of the Medical Sciences, vol. 330, no. 6, pp. 269-272, 2005.

[80] Z. Ali, C. S. Ulrik, T. Agner, and S. F. Thomsen, "Association between atopic dermatitis and the metabolic syndrome: a 
systematic review," Dermatology, vol. 234, no. 3-4, pp. 79-85, 2018.

[81] D. Q. Xiao, P. H. Xu, H. Deng et al., "The characterization and clinical significance of PI3K/Akt signaling pathway activation in the peripheral $\mathrm{T}$ cells of pediatric patients with atopic dermatitis," International Journal of Clinical and Experimental Medicine, vol. 10, pp. 2904-2910, 2017.

[82] B. Hartmann, R. Riedel, K. Jörß et al., "Vitamin D receptor activation improves allergen-triggered eczema in mice," Journal of Investigative Dermatology, vol. 132, no. 2, pp. 330-336, 2012.

[83] H. A. Ahmed and I. Y. Alkali, "In silico molecular docking studies of some phytochemicals against peroxisome-proliferator activated receptor gamma (PPAR- $\gamma$ )," GSC Biological and Pharmaceutical Sciences, vol. 5, no. 2, pp. 1-5, 2018.

[84] L. Alappat, M. Valerio, and A. B. Awad, "Effect of vitamin D and $\beta$-sitosterol on immune function of macrophages," International Immunopharmacology, vol. 10, no. 11, pp. 1390$1396,2010$.

[85] M. Megna, M. Napolitano, C. Patruno et al., "Systemic treatment of adult atopic dermatitis: a review," Dermatology and Therapy, vol. 7, no. 1, pp. 1-23, 2017.

[86] X. Zhou, S. W. Seto, D. Chang et al., "Synergistic effects of Chinese herbal medicine: a comprehensive review of methodology and current research," Frontiers in Pharmacology, vol. 7, p. 201, 2016.

[87] J. Dong, "The relationship between traditional Chinese medicine and modern medicine," Evidence-Based Complementary and Alternative Medicine, vol. 2013, Article ID 153148, 10 pages, 2013. 